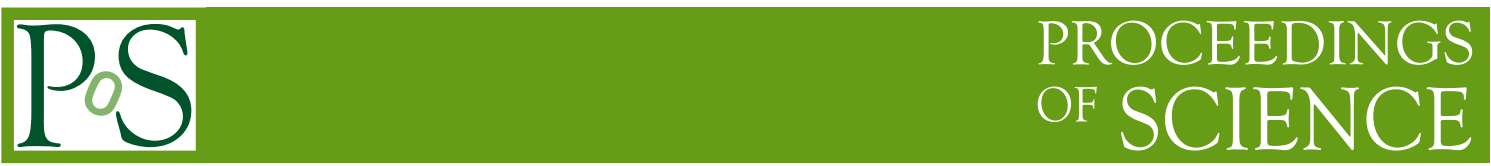

\title{
Neutrino Oscillation Present: Session Summary
}

\author{
Camillo Mariani \\ Center for Neutrino Physics, Virginia Tech, Blacksburg, VA, 24061 \\ E-mail: marianievt.edu

\section{Mariam Tórtola} \\ AHEP Group, Institut de Física Corpuscular-C.S.I.C./Universitat de València. \\ Parc Cientific de Paterna. Catedràtic José Beltrán, 2. E-46980 Paterna (València) - SPAIN \\ E-mail: mariameific.uv.es
}

\begin{abstract}
Here we summarize the talks given in the parallel session I, "Neutrino Oscillations: Present" at the Neutrino Oscillation Workshop 2016. The session included talks on the latest results from the reactor experiments Daya Bay, Double Chooz and SOLID, the accelerator experiment OPERA and MicroBooNE, a review of the current and future measurements of the Borexino detector and a review on current challenges to understand the reactor neutrino spectrum and the importance of nuclear models and cross section on accelerator neutrino experiments. On the theoretical side, the contributed talks discussed the latest developments on the standard model of the Sun, the most recent oscillation parameter determinations from global fits of neutrino data, as well as a new formalism to describe three-neutrino oscillations. The session also included talks on the impact of new physics beyond the Standard Model, such as non-standard neutrino interactions or non-unitary mixing, on the determination of neutrino oscillation parameters.
\end{abstract}

Neutrino Oscillation Workshop

4 - 11 September, 2016

Otranto (Lecce, Italy) 


\section{Introduction}

In the last few years there has been a tremendous progress in neutrino oscillation physics. Indeed, the neutrino oscillation phenomenon has been observed in a rich variety of experiments with increasing precision, allowing a very accurate determination of the oscillation parameters in a three-neutrino framework $[1,2,3]$. Reactor neutrino experiments have reached phenomenal uncertainties and now the $\theta_{13}$ mixing angle is one of the best measured quantities. High precision came at a cost: new puzzles emerged in the big picture, the reactor anomaly has been confirmed together with interesting features in the measured reactor anti-neutrino spectrum. New short baseline experiments, both at reactors and accelerators, are now taking data or are being prepared and they will shine light on the existence of a fourth sterile neutrino. SOLID is pretty close to a data taking and the MicroBooNE experiment is taking data in the BNB and it is making tremendous progress in the automatic reconstruction of neutrino events in liquid argon. Borexino has made wonderful discovery on solar neutrinos and it is now starting a new phase with incredible purity and low background contamination.

Nowadays, the most important missing piece of information in the neutrino sector is the measurement of CP violation. To this aim, several accelerator experiments such as DUNE or T2HK have been proposed to study neutrino properties over long distances in combination with underground detectors. A great challenge for these experiments is to be able to achieve low uncertainties and that poses new challenges for the neutrino theory community. Nuclear physics is becoming once more prominent in understanding and describing neutrino physics. The new generation of neutrino experiments will also be crucial to probe neutrino properties beyond the Standard Model, as for example, non-standard neutrino interactions [4] or unitarity violation in the neutrino mixing matrix due to the admixture with heavy fermion messengers [5]. Both types of new physics are directly associated to neutrino mass generation and therefore might be decisive in elucidating the origin of neutrino mass.

\section{Experimental Contributions}

The Daya Bay (DB) and Double Chooz (DC) Collaborations focus their effort to measure the $\theta_{13}$ neutrino mixing angle using the inverse beta decay (IBD) channel $\left(\bar{v}_{e}+p \rightarrow e^{+}+n\right)$ to search for oscillations $\bar{v}_{e} \rightarrow \bar{v}_{e}$. The DB collaboration [6] presented analysis involving 3 underground experimental halls, 8 anti-neutrino detectors summing to a total target mass of $160 \mathrm{t}$ exposed to 6 nuclear reactors for a total power of $17.4 \mathrm{GW}_{t h}$. The near site experimental halls are located respectively at $363 \mathrm{~m}$ and at $\sim 500 \mathrm{~m}$ from the reactors. The far detector hall is located at $1,910 \mathrm{~km}$ and at $1,510 \mathrm{~km}$ from the reactor sites. Daya Bay presented [6] results for the Gd analysis: $\sin ^{2} \theta_{13}=(8.41 \pm 0.27$ (stat. $) \pm 0.19$ (syst. $\left.)\right) \times 10^{-2}$ [6] with a $\left|\Delta m_{e e}^{2}\right|=(2.50 \pm 0.06$ (stat. $) \pm$ $0.06($ syst. $)) \times 10^{-3} \mathrm{eV}^{2}$ [6] obtained using 1,230 days of data. The DC collaboration presented [7] a two detector analysis that gives a $\sin ^{2} \theta_{13}=0.111 \pm 0.018$ (stat. + syst.) [7], where the error is limited by statistics and it was obtained using 9 months of data. There is no hint of a light sterile neutrino observed and DB set a more stringent limit for $\left|\Delta m_{41}^{2}\right|<0.2 \mathrm{eV}^{2}$ and it was also presented a combined analysis of $\bar{v}_{e}$ with the Bugey experiment and with the MINOS $\bar{v}_{\mu}$ disappearance experiment. 
Dwyer presented a new analysis [9] focus on the spectral distortions around the 4-6 MeV energy region and concluded that the significant differences that are seen by experiments are due to missing data, biased branching fractions and shape corrections for 8 particular branching fractions that specifically contributes to the 4-6 MeV energy region.

The SOLID experiment [10] is searching for short baseline oscillation, light sterile neutrinos, direct and precise measurement of the ${ }^{235} \mathrm{U} \bar{v}_{e}$ spectrum and insight for reactor neutrino flux model in the 4-6 MeV region. SOLID is capable of high energy and spatial resolutions, it is made of an homogeneous medium that can be easily inter-calibrated and is very effective in background rejection making it very suitable for non-proliferation applications as well. The final SOLID detector will be $2 \mathrm{t}$ fiducial volume and it will be deployed by the end of 2017 between 5.5 and $12 \mathrm{~m}$ from $\mathrm{BR} 2$ reactor in Belgium.

The OPERA experiment [11] was designed to directly observe, for the first time in appearance mode, the oscillation $v_{\mu} \rightarrow v_{\tau}$ in a pure $v_{\mu}$ beam produced by the CNGS. There have been 5 candidates observed so far. The expected number of signal events is $2.64 \pm 0.53$ with a total background of $0.25 \pm 0.05$. OPERA also searched for $v_{\mu} \rightarrow v_{e}$ oscillation, they observed events (34) is in agreement with the expected background plus standard oscillations. OPERA $v_{\tau}$ and $v_{e}$ appearance results have been used to derive limits on the mixing parameters of massive sterile neutrinos, at large $\Delta m_{41}^{2}: \sin ^{2}\left(2 \theta_{\mu \tau}\right)<0.119$ at $90 \%$ CL [12] and for non-standard interactions they obtained $\sin ^{2}\left(2 \theta_{\text {new }}\right)<7.2 \times 10^{-3}$ at $90 \%$ CL [13].

The Borexino experiment started in 2007 and released an extraordinary amount of results [14]. The new phase of the project is on-going and a new project SOX will start in 2017 [18]. Borexino is an ultra pure scintillator $(270 \mathrm{t})$ sphere surrounded by a buffer region, a water tank and a stainless steel supporting structure. The scintillation light ( $\sim 500$ photon-electron/MeV) coming from a neutrino interaction is readout by photomultiplier tubes that reconstruct the energy (5\%@1 MeV) and position (10 cm@1 MeV) of the signals and provide pulse shape capability. Borexino provided the first detection of pp and pep neutrino from the sun [15, 16]. After completing the detector insulation in 2015 they are preparing to measure the CNO- $v$ in the next few years. Borexino also published results on geo-neutrinos using IBD events. Their latest analysis [17] is based on 2056 days of data and gives the following fluxes: $\Phi(U)=(2.7 \pm 0.7) \times 10^{6} \mathrm{~cm}^{-2} \mathrm{~s}^{-1}$ and $\Phi(T h)=$ $(2.3 \pm 0.6) \times 10^{6} \mathrm{~cm}^{-2} \mathrm{~s}^{-1}$.

Nieves reviewed recent results and open questions in the physics of neutrino cross-sections [19]. There exist dedicated experiments at Fermilab, Minerva and MicroBooNE [20] which seeks to measure low energy neutrino interactions in various mediums and try to describe pion production in nuclei. Understanding pion and meson exchange production is important because in quasi-elastic (QE) interactions (mostly used by experiment due to the simple topology), if a pion is misidentified or absorbed, then a non-QE event can be identified as QE, making the determination of the neutrino energy wrong [21].

Furmanski [20] presented the MicroBooNE experiment in the more general context of the short baseline program at Fermilab and the unprecedented sensitivity to $\mathrm{eV}$-scale sterile neutrino searches [23]. Furmanski presented a Michel electron spectrum using stopping-and-decaying cosmic muons. The detector has been collecting neutrino data since October 2015 and they have now collected $3.57 \times 10^{20}$ POT. They are able to select neutrino events based on beam timing and fully reconstruct neutrino candidate events. They presented preliminary results on charged current inclu- 
sive neutrino selection and on charged current $\pi^{0}$ production. They have a rich program of neutrino cross section measurements that should be published in the next few years.

\section{Theoretical Contributions}

The analysis of neutrino data from solar experiments is a powerful tool to study neutrino oscillations as well as other neutrino properties. To this purpose one needs to rely on the Standard Solar Model (SSM), that predicts the out-coming solar neutrino fluxes. In this parallel session, Serenelli presented a new generation of SSMs including the latest improvements in the field [24]. These models, denoted as Barcelona 16 (B16), introduce several updates in the physical inputs considered, such as the solar composition or the nuclear reaction rates, updated to the latest theoretical and experimental developments. They also introduce improved treatments of the equation of state and radiative opacities, with more generous estimates on the uncertainties. One of the main results of the new SSMs, however, is that they produce very small changes in the helioseismic quantities. Consequently, the discrepancy between low metallicity solar models and current helioseismic measurements, as for instance the sound speed or density profiles, (known as the solar abundance problem) remains. The role of the $\mathrm{CN}$ solar neutrino fluxes, very relevant to probe the solar core composition, was also discussed.

Capozzi presented an update on the status of three-neutrino oscillation parameters considering all relevant neutrino data released after the summer of 2016 [25]. This includes data from all solar neutrino experiments, reactor data from KamLAND as well as from the short-baseline reactor experiments Daya Bay, RENO and Double Chooz. Atmospheric data from Super-Kamiokande and IceCube-DeepCore are also considered, together with long-baseline accelerator data from MINOS, $\mathrm{T} 2 \mathrm{~K}$ and NOvA. As it was shown, the combination of all data sets allows a very accurate determination of neutrino oscillation parameters. However, the main unknowns in the three-neutrino oscillation scenario remain unsolved. One example is the precise value of the atmospheric mixing angle. There are improvements in its determination, and indeed the latest long-baseline results disfavor maximal mixing at $2 \sigma$, but the ambiguity on its octant is still present. While the shortbaseline reactor measurements of $\theta_{13}$ imply a different octant preference for normal and inverted neutrino mass hierarchy, the analysis of atmospheric data moves the best fit point to the first octant for both hierarchies. As a result, the global fit preference for the first octant is not conclusive, specially for inverted hierarchy. Regarding CP violation, a hint for a non-zero CP phase at $2 \sigma$ is obtained, with values of $\delta_{C P}=\pi / 2$ disfavored at $3 \sigma$. The effect of neutrino oscillation data on the value of the absolute neutrino mass was also discussed. The combination of neutrino oscillation data with results from cosmology and neutrinoless double beta decay experiments improves the determination of the sum of neutrino masses $\left(\sum=m_{1}+m_{2}+m_{3}\right)$ and the effective neutrino mass for neutrinoless double beta decay, $m_{\beta \beta}$. Also in the standard three-neutrino scenario, Hong-Jian He presented the connection between the leptonic unitarity triangle (LUT) and the formalism of neutrino oscillations [27]. The expression for the neutrino conversion probability in vacuum as well as its geometrical expression in terms of the LUT was presented. It is interesting to notice that, in this formalism, the angles of the LUT act as phase shifts in neutrino oscillations. More remarkably, within this parameterization, neutrino oscillations in a given appearance channel can be described by only three independent parameters, in principle different for each channel. This 
fact allows the direct measurement of LUT parameters from a given neutrino conversion channel. The leptonic unitarity triangle formulation can also be applied to neutrino oscillations in matter by defining an effective LUT. Thanks to the hierarchical relation between the solar and atmospheric neutrino mass splittings, a perturbative expansion around the ratio of these mass splittings allows a simplified expression for the neutrino oscillation probability in matter. For a unique neutrino oscillation channel, the derived formula is more compact than the standard formula obtained from the three-neutrino mixing matrix.

Besides the standard three-flavor scenario for neutrino oscillations, in this parallel session there were two presentations considering new physics scenarios beyond the Standard Model.

Forero presented a talk about the current status of non-standard neutrino interaction searches at current facilities [26]. Non-standard interactions (NSI) of neutrinos with matter have been studied in different contexts. However, current bounds on some of the NSI couplings are not very strong, leaving plenty of room for them to show up or being further constrained at present experiments. In the first part of the talk, the role of NSI in the production and detection of reactor antineutrinos in Daya Bay was discussed. Using the latest data from Daya Bay, independent bounds on the NSI couplings were derived. It was also shown that, in some cases, the robustness of the $\theta_{13}$ determination in Daya Bay can be severely affected by the presence of these interactions. NSI can also be probed in long-baseline accelerator experiments. The possible degeneracy between the standard CP violating phase and additional sources of CP violation coming from NSI was discussed in the context of $\mathrm{T} 2 \mathrm{~K}$ and NOvA. The main conclusion from this analysis is that, in the presence of NSI, it would not be possible to disentangle the origin of the potentially observed CP violation.

Miranda considered in his talk the possibility of a non-unitary neutrino mixing matrix [28]. This scenario is theoretically motivated from models of neutrino masses with extra heavy states. In this case, the $3 \times 3$ light neutrino mixing matrix will not be unitary in general. The speaker presented a general parameterization for non-unitarity that turns out to be very useful for the study of neutrino oscillations in this scenario, since it factorizes out in terms on the standard threeneutrino mixing matrix and a triangular matrix with three real and three complex entries. The new non-unitarity parameters might be constrained from current lepton universality and neutrino oscillation data. Similarly to the NSI case discussed above, the presence of non-unitarity may give rise to degeneracies in the determination of the standard CP violating phase, $\delta$.

\section{References}

[1] F. Capozzi, E. Lisi, A. Marrone, D. Montanino and A. Palazzo, Nucl. Phys. B 908 (2016) 218 doi:10.1016/j.nuclphysb.2016.02.016 [arXiv:1601.07777 [hep-ph]].

[2] D. V. Forero, M. Tortola and J. W. F. Valle, Phys. Rev. D 90 (2014) no.9, 093006 doi:10.1103/PhysRevD.90.093006 [arXiv:1405.7540 [hep-ph]].

[3] I. Esteban, M. C. Gonzalez-Garcia, M. Maltoni, I. Martinez-Soler and T. Schwetz, arXiv:1611.01514 [hep-ph].

[4] O. G. Miranda and H. Nunokawa, New J. Phys. 17 (2015) no.9, 095002 doi:10.1088/1367-2630/17/9/095002 [arXiv:1505.06254 [hep-ph]]. 
[5] F. J. Escrihuela, D. V. Forero, O. G. Miranda, M. Tortola and J. W. F. Valle, Phys. Rev. D 92 (2015) no.5, 053009 Erratum: [Phys. Rev. D 93 (2016) no.11, 119905] doi:10.1103/PhysRevD.93.119905, 10.1103/PhysRevD.92.053009 [arXiv:1503.08879 [hep-ph]].

[6] V. Pek, Daya Bay results, talk given at NOW2016. These proceedings (2016).

[7] C. Buck, Double Chooz results, talk given at NOW2016. These proceedings (2016).

[8] F. P. An et al. [Daya Bay Collaboration], Phys. Rev. Lett. 117, no. 15, 151802 (2016) doi:10.1103/PhysRevLett.117.151802 [arXiv:1607.01174 [hep-ex]].

[9] D. Dwyer, Reactor Antineutrinos: il buono, il brutto, il cattivo, talk given at NOW2016. These proceedings (2016).

[10] B. Guillon, SOLID results, talk given at NOW2016. These proceedings (2016).

[11] G. Galati, Neutrino oscillation search with the OPERA experiment, talk given at NOW2016. These proceedings (2016).

[12] N. Agafonova et al. [OPERA Collaboration], JHEP 1506, 069 (2015) doi:10.1007/JHEP06(2015)069 [arXiv:1503.01876 [hep-ex]].

[13] N. Agafonova et al. [OPERA Collaboration], JHEP 1307, 004 (2013) Addendum: [JHEP 1307, 085 (2013)] doi:10.1007/JHEP07(2013)004, 10.1007/JHEP07(2013)085 [arXiv:1303.3953 [hep-ex]].

[14] S. Zavatarelli, Recent solar and geo- $v$ results from Borexino, talk given at NOW2016. These proceedings (2016).

[15] G. Bellini et al. [BOREXINO Collaboration], Nature 512, no. 7515, 383 (2014). doi:10.1038/nature 13702

[16] G. Bellini et al. [Borexino Collaboration], Phys. Rev. Lett. 108, 051302 (2012) doi:10.1103/PhysRevLett.108.051302 [arXiv:1110.3230 [hep-ex]].

[17] M. Agostini et al. [Borexino Collaboration], Phys. Rev. D 92, no. 3, 031101 (2015) doi:10.1103/PhysRevD.92.031101 [arXiv:1506.04610 [hep-ex]].

[18] S. Ranucci, SOX and light sterile neutrinos, talk given at NOW2016. These proceedings (2016).

[19] J. Nieves, Qe-like scattering and neutrino energy reconstruction, talk given at NOW2016. These proceedings (2016).

[20] A. Furmanski, Recent MicroBooNE results, talk given at NOW2016. These proceedings (2016).

[21] A. M. Ankowski and C. Mariani, arXiv:1609.00258 [hep-ph].

[22] O. Benhar, P. Huber, C. Mariani and D. Meloni, arXiv:1501.06448 [nucl-th].

[23] G. Raselli, Sterile searches with Liquid Argon at FNAL, talk given at NOW2016. These proceedings (2016).

[24] A. Serenelli, Solar Models and Neutrinos: What's New Under the Sun?, talk given at NOW2016. These proceedings (2016).

[25] F. Capozzi, Status of three-neutrino mixing, talk given at NOW2016. These proceedings (2016).

[26] D. V. Forero, (Non)standard oscillations at current facilities, talk given at NOW2016. These proceedings (2016).

[27] H-J. He, Leptonic unitarity triangle, neutrino oscillation and CP violation, talk given at NOW2016. These proceedings (2016).

[28] O. G. Miranda, Nonunitary mixing: current constraints and new ambiguity, talk given at NOW2016. These proceedings (2016). 